\title{
Método MAIN para planificar, aplicar y divulgar la innovación educativa
}

\section{MAIN Method for Planning, Applying and Disseminating Educational Innovation}

Ángel Fidalgo-Blanco

Laboratorio de Innovación en Tecnologías de la Información. LITI. Universidad Politécnica de Madrid.

Calle de Ríos Rosas 21, 28003 Madrid. España

angel.fidalgo@upm.es

ORCID: https://orcid.org/0000-0003-4034-7757

María Luisa Sein-Echaluce

Departamento de Matemática Aplicada, Universidad de Zaragoza, Campus Rio Ebro,

Calle de María de Luna 3, 50018 Zaragoza, España

mlsein@unizar.es

ORCID: https://orcid.org/0000-0002-6873-0996

\section{Resumen}

La innovación educativa es un proceso creativo cuyo principal objetivo es mejorar aspectos relacionados con el aprendizaje. Por tanto, proporciona una solución a determinados problemas o produce mejoras significativas en el aprendizaje. No obstante, el carácter creativo del proceso de innovación hace difícil la planificación de cualquier experiencia innovadora, prever sus resultados, seguir un método de aplicación eficaz y eficiente e incluso transferirla. Para paliar esta situación, el método MAIN (Método de Aplicación de la INnovación educativa) se ha desarrollado como método de diseño de la innovación educativa que permite su planificación, aplicación y divulgación. El objetivo de este trabajo es presentar este método como un conjunto de pasos estructurados que permiten tener una visión previa de: el método a utilizar, el esfuerzo que necesitará, la tecnología que la apoyará y la previsión de su impacto. También facilita la identificación de actividades a realizar, de tal forma que sirve de guía para la aplicación de la innovación educativa en una asignatura concreta. Se presentan los distintos módulos del método MAIN aplicados a una situación concreta (pasividad del alumnado), con un método de innovación educativa que es actualmente tendencia (Flip Teaching) y con una estrategia de aplicación que permite divulgar, de forma científica, la innovación realizada.

\section{Palabras Clave}

Innovación educativa; Tecnología educativa; Métodos educativos; Aprendizaje activo; Clase invertida

\section{Abstract}

Educational innovation is a creative process whose aim is to improve aspects related to learning. Therefore it provides a solution to certain problems or produces significant learning improvements. However, the creative nature of the innovation process makes it difficult to plan any innovative experience, foresee its results, follow an efficient and effective application method and even transfer it. To alleviate this situation, the method MAIN (Method for Applying Innovation in educatioN) has been developed as a design method of educational innovation to allow its planning, application and dissemination. The objective of this work is to present this method as a set of structured steps that provides a preview of: the method to be used, the effort that will be needed, the technology that will support it and the forecast of its impact. It also facilitates the identification of activities to be carried out, in such a way that they serve as a guide for the application of educational innovation in a specific subject. The different modules of the MAIN method applied to a specific situation (passivity of students) are presented, with an educational innovation method that is currently a trend (Flip Teaching) and with an application strategy that allows to disseminate, in a scientific way, the innovation which has been done.

\section{Keywords}

Educational Innovation; Educational Technology; Educational Methods; Active Learning; Flip Teaching 


\section{Introducción}

La innovación educativa y la innovación, en general, se suele relacionar con procesos creativos, de emprendimiento, e incluso a estados emocionales. Así mismo, las definiciones de innovación educativa suelen estar relacionadas con las palabras cambio y mejora (Sein-Echaluce, Fidalgo-Blanco, \& Alves, 2017).

De esa forma se comprende el fin de la innovación educativa: cambiar y mejorar. Sin embargo, el proceso de realizar y validar la innovación no suele asociarse a indicadores, planificación, previsión de resultados y gestión.

En el lado opuesto se encuentra la visión de la ingeniería. Esta se entiende como una aplicación proceso que aporta soluciones a problemas y necesidades concretas, a través del desarrollo de productos y servicios. La ingeniería aplica conocimientos, métodos y tecnologías de forma óptima para solucionar los problemas a partir de la identificación de necesidades. La aplicación está perfectamente definida, los procesos y métodos también lo están.

Aunque la innovación educativa se vea como algo etéreo, abstracto, indefinido e imprevisible y la ingeniería como algo medible, concreto, definible y previsible, ambas ciencias comparten objetivos: buscar soluciones y mejoras a través de cambios planificados. Así pues, cabe preguntarnos ¿se puede unir la innovación educativa con procesos y métodos de ingeniería? Si esto fuese posible, tendríamos una forma planificada, medible, previsible y gestionable de aplicar la innovación educativa, sin perder la creatividad, las emociones (tan necesarias para que se produzca un aprendizaje efectivo) y la novedad.

Uno de los principales problemas de la innovación educativa es que el profesorado no tiene guías o pautas para planificarla, aplicarla y medirla. Esta situación lleva a que los centros educativos, en concreto las universidades, no tengan criterios para identificar la innovación educativa, ni para clasificarla, organizarla y distribuirla en los procesos educativos donde es necesaria. Estefactor también es importante pues supone una dificultad por parte de las instituciones académicas (regionales, nacionales e internacionales) para llevar a cabo el reconocimiento del trabajo de innovación realizado por el profesorado.

A pesar de todo ello, es cada vez más numeroso el profesorado que innova, que comparte su experiencia a través de la Web 2.0 y que organiza encuentros tanto físicos como virtuales. Pero debido a esa visión abstracta de la innovación educativa, no se ha conseguido unificar las experiencias de innovación educativa (ni al profesorado innovador) para que, con su acción, se produzca un efecto 
transformador en la educación.

Esta situación justifica que se integre la visión de los procesos de ingeniería con la visión de los procesos de innovación educativa (García-Peñalvo, 2015), ya que abre las puertas a que la innovación educativa se pueda planificar, medir, clasificar, organizar y a que, principalmente, tenga un impacto transformador en el modelo educativo (Fidalgo-Blanco, Sein-Echaluce, \& García-Peñalvo, 2014 \& 2015). Esta integración ya se ha realizado en otros cambios similares, por ejemplo, en la creación de conocimiento y su gestión para crear valor dentro de una empresa. Uno de estos métodos (Nonaka \& Takeuchi, 1995) se basa en integrar las acciones de fuerza creativa (similares a los procesos de innovación) con los burocráticos (procesos planificados y estructurados para alcanzar un objetivo de forma óptima).

Desde grupos de investigación de la Universidad Politécnica de Madrid, de la Universidad de Zaragoza y de la Universidad de Salamanca se viene desarrollando un método que permite planificar la innovación educativa, valorar el esfuerzo que conlleva su aplicación, prever los resultados, seleccionar las tecnologías más adecuadas a cada situación y transmitir los resultados y la experiencia en sí misma, a través de todo el tejido del sistema educativo.

El método se denomina MAIN (Método de Aplicación de la INnovación educativa - Method for Applying Innovation in educatioN) (Fidalgo-Blanco, Sein-Echaluce, \& García-Peñalvo, 2018a). Su objetivo es disponer de una herramienta que ofrezca una visión realista de la innovación educativa que se va a realizar. MAIN permite:

- Identificar la situación de aprendizaje que se quiere mejorar.

- Identificar las innovaciones más adecuadas (e incluso diseñar nuevas).

- Identificar y relacionar las actividades que debe realizar tanto el profesorado como el alumnado.

- Identificar la funcionalidad y misión de la tecnología a utilizar.

- Identificar el esfuerzo y habilidades tecnológicas necesarias (tanto para el profesorado como por parte del alumnado).

- Ofrecer de forma independiente la innovación educativa y la innovación tecnológica.

- Aplicar múltiples estrategias: para la divulgación científica, para cambiar el rol del alumnado y del profesorado y para la transferibilidad de la experiencia acumulada (a cualquier asignatura) y divulgación científica.

- Prever los resultados y el impacto. 
MAIN trabaja con modelos de innovación educativa consolidados, también con los que son tendencia, e incluso permite organizar cualquier innovación futura.

El método está estructurado en tres módulos relacionados entre sí, pero que se pueden aplicar de forma independiente en cualquier área de conocimiento y contexto de aprendizaje. Cada módulo se corresponde con una etapa crítica del proceso de innovación educativa: planificación, aplicación y divulgación.

En el siguiente apartado se presenta el modelo conceptual de MAIN para, a continuación, incluir un ejemplo concreto teniendo a la vista una tendencia actual de innovación educativa: Flip Teaching o Flipped Classroom.

\section{Método}

Como se ha dicho, el modelo se compone de tres módulos conceptuales que se corresponden con las etapas que todo profesorado tiene que tener en cuenta antes de realizar una experiencia de innovación educativa: planificación, aplicación y divulgación.

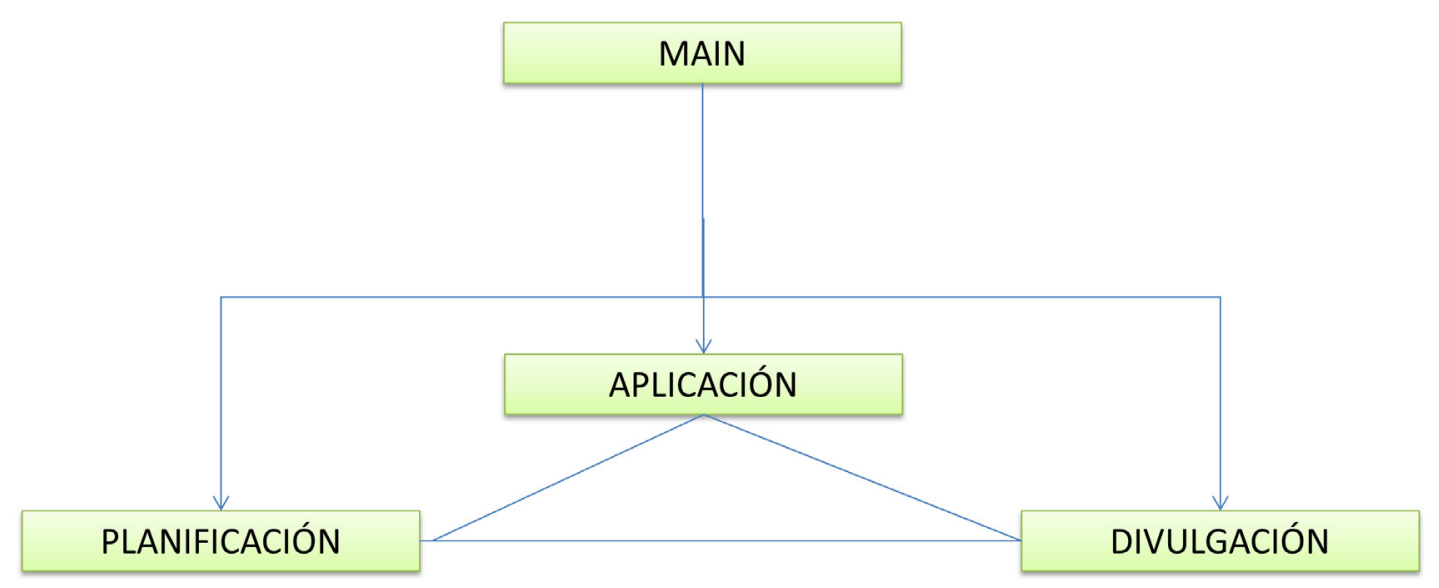

Figura 1. Método MAIN

\subsection{Módulo Planificación}

El objetivo de este módulo es identificar el contexto donde se va a realizar la innovación y los elementos necesarios para realizar dicha innovación educativa. Sus principales sub-módulos: situación de aprendizaje, público objetivo, método de innovación a utilizar e impacto, se muestran en la Figura 2 y se explican a continuación. 


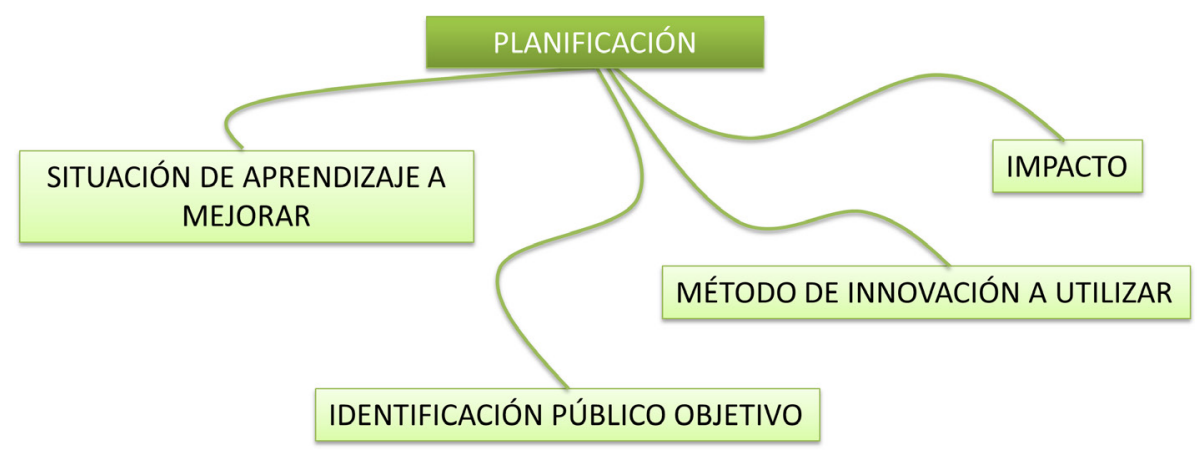

Figura 2. Módulo de planificación del método MAIN

\subsubsection{Situación de aprendizaje}

La situación de aprendizaje a mejorar identifica las problemáticas actuales más habituales que tienen incidencia de forma negativa en el aprendizaje. A modo de ejemplo:

- Alumnado con conocimientos previos distintos o insuficientes para adquirir un concepto, destreza, habilidad o competencia.

- Alumnado inactivo durante el proceso de aprendizaje tanto dentro del aula como fuera del aula.

Todas estas situaciones están relacionadas con aspectos emocionales -como la motivación, el interés y la curiosidad-, con el fracaso escolar e incluso con competencias genéricas.

\subsubsection{Público objetivo}

Hace referencia a las características del alumnado al que va dirigida la acción. En ocasiones será todo el alumnado de una determinada asignatura (en cuanto, por ejemplo, a la participación activa); en otras estará definido por una serie de características (alumnos a los que les falten conocimientos previos).

\subsubsection{Método de innovación a utilizar}

En este sub-módulo se tiende a no reinventar la rueda. Se presentan modelos de innovación educativa, validados y contrastados, dirigidos al tipo de público objetivo, que consigan mejoras en la misma situación de aprendizaje identificada. El profesorado, el centro o la institución pueden elegir un modelo u otro en base al interés, estrategia o tecnología implantada. Este sub-módulo también se puede utilizar para crear un nuevo método de innovación (si ninguno de los identificados es válido). 


\subsubsection{Impacto}

En este sub-módulo se pueden identificar tanto indicadores para medir el resultado de la innovación (indicadores habitualmente asociados a las personas: motivación, cooperación, creación de conocimiento, etc.) como al impacto en los resultados de aprendizaje (asociado a contenidos o a resultados académicos).

\subsection{Módulo Aplicación}

La principal misión del módulo Aplicación es hacer de guía tanto en la preparación como en la aplicación del propio proceso de innovación educativa. Este módulo se divide en tres sub-módulos: modelo funcional, tecnología y estrategia. Estos sub-módulos permiten identificar las actividades a realizar, los elementos con los que se trabaja en cada actividad (personas, contenidos y tecnologías), la misión de la tecnología, los criterios para elegir la tecnología y los cambios de rol asociados tanto al profesorado como al alumnado. Se explica a continuación.

\subsubsection{Modelo funcional}

Los componentes del modelo son procesos relacionados entre sí. Los procesos pueden ser conceptuales (expresan la idea del método de innovación educativa elegido) o funcionales (agrupan un conjunto de actividades que el alumnado y profesorado necesitan realizar para alcanzar un determinado objetivo). Para cada modelo funcional también se identifica la misión de la tecnología que se utilizará en cada componente funcional y que apoyará las acciones del profesorado y del alumnado. El modelo funcional permite conocer el impacto que tendrá la innovación educativa en el contexto de aprendizaje, valorar el esfuerzo que nos llevará la inclusión de esa innovación en nuestro contexto y ser consciente de los cambios metodológicos a realizar.

\subsubsection{Tecnología}

Este sub-módulo consiste en la recopilación de las distintas tecnologías que presentan la funcionalidad (o misión) especificada en el proceso funcional. La idea principal es disponer de un conjunto de tecnologías posibles para realizar las distintas actividades. Esta identificación permite al profesorado elegir la tecnología más adecuada en base a sus propios criterios (facilidad de uso, disponibilidad, experiencia, etc.). Otro aspecto singular es que al tener identificada la funcionalidad (o misión) de la tecnología, se puede sustituir una por otra sin que se altere el modelo. Esto es importante, ya que muchas experiencias de innovación educativa se asocian a la tecnología y cuando esta queda obsoleta sucede lo mismos con la innovación educativa realizada. 


\subsubsection{Estrategia "cambio de rol"}

Un mismo modelo educativo con una misma tecnología puede resultar un éxito o un fracaso, dependerá de la estrategia con la que utilicemos ambos. En algunas ocasiones la experiencia de innovación educativa fracasa porque el alumnado tiene una serie de hábitos adquiridos y consolidados (por ejemplo, no ir a tutorías, estudiar unos días antes del examen, no cooperar, etc.). Es muy importante que se identifiquen los hábitos adquiridos y, en base a los mismos, se aplique un plan de acción para introducir los nuevos hábitos. La estrategia para el cambio de hábitos exige nuevos roles tanto del profesorado como del alumnado.

\subsection{Módulo de divulgación}

Difundir la innovación educativa es importante para producir un impacto en las instituciones y en el modelo educativo. Para poder realizar una divulgación eficaz y eficiente es necesario integrar la forma de realizar la innovación educativa, los mecanismos de gestión de la innovación que permitan la transferibilidad, el compromiso institucional y la cooperación del profesorado. El método MAIN trabaja con dos tipos de divulgación, una muy consolidada como es la divulgación científica (que suele trabajar con los resultados y su validación) y otra incipiente que es la transferibilidad de la innovación (que suele trabajar con el impacto en los procesos de aprendizaje y la experiencia adquirida por el profesorado al realizar la innovación). A continuación se desarrollan ambos tipos de divulgación.

\subsubsection{Divulgación científica}

Para el profesorado es importante divulgar el trabajo en congresos y revistas científicas. Para ello se debe plantear la investigación a través del conocimiento del estado del arte, análisis científico de evidencias y utilización de herramientas de medición validadas. Este tipo de divulgación está estandarizado: el profesorado debe utilizar métodos científicos en la realización de la innovación educativa, bien creando nuevas innovaciones o aplicando las existentes (Boyer, 1990; RodríguezConde et al., 2016). Las labores de identificación y selección de experiencias de innovación educativa de calidad científica recaen habitualmente en los comités científicos de congresos y revistas. En este caso, por tanto, la divulgación se realiza a través de actas, libros y artículos y sus indicadores de calidad tienen repercusión en el sistema educativo pero como referentes en el campo de la investigación, no en el cambio del modelo educativo. 


\subsubsection{Transferibilidad de la innovación}

Una buena práctica de innovación educativa lo es, no solamente por su repercusión en la mejora de los resultados (eficacia), sino por su eficiencia, sostenibilidad y transferibilidad. Esta última característica significa que la innovación educativa realizada en una asignatura se debe poder transferir a cualquier otra asignatura, aunque no sea de la misma área de conocimiento o nivel educativo. Esta característica es clave para conseguir un impacto transformador en el modelo educativo de un centro de formación determinado y, como consecuencia, en el modelo educativo a nivel global. Este tipo de divulgación es poco conocida y se basa en identificar y medir el impacto en los procesos educativos, perfiles de alumnado y contextos de aprendizaje concretos.

\section{Un caso concreto de aplicación del método MAIN y sus resultados}

Se presenta en esta sección un ejemplo del método MAIN para un caso concreto que entendemos de interés general entre el profesorado. Dicho caso se imparte actualmente en cursos y talleres en varias universidades españolas (Universidad de la Coruña, Universidad de León, Universidad de Salamanca, Universidad de Vigo, Universidad de Zaragoza y Universidad Politécnica de Madrid). Comenzaremos aplicando los tres módulos que componen el método MAIN para este caso: módulo de planificación, módulo de aplicación y módulo de divulgación.

\subsection{Módulo de planificación}

La tabla 1 muestra, de forma esquemática, los cuatro sub-módulos que componen el módulo de planificación y que desarrollaremos a continuación.

\begin{tabular}{|c|c|c|c|}
\hline $\begin{array}{l}\text { Situación de } \\
\text { aprendizaje }\end{array}$ & $\begin{array}{c}\text { Métodos de } \\
\text { innovación educativa }\end{array}$ & Público objetivo & Impacto \\
\hline \multirow{5}{*}{$\begin{array}{l}\text { Alumnado } \\
\text { inactivo }\end{array}$} & Inteligencia colectiva & \multirow{4}{*}{$\begin{array}{l}\text { Alumnado en } \\
\text { situaciones de } \\
\text { aprendizaje } \\
\text { teórico } \\
\text { (por ejemplo, } \\
\text { lecciones } \\
\text { magistrales) }\end{array}$} & \\
\hline & Micro Flip Teaching & & $\begin{array}{l}\text { Aumento de: preguntas, debates, reflexiones, } \\
\text { conocimiento (creación, cooperación y transferencia) y } \\
\text { asistencia a clase voluntaria } \\
\text { Mejora de resultados académicos en conocimientos } \\
\text { teóricos } \\
\text { Mejora de la interacción estudiante - estudiante } \\
\text { Validez de los recursos creados por el alumnado } \\
\text { Impacto de la retroalimentación en mejoras de } \\
\text { aprendizaje en conocimientos complejos }\end{array}$ \\
\hline & $\begin{array}{l}\text { Aprendizaje basado } \\
\text { en retos }\end{array}$ & & \\
\hline & Aprendizaje servicio & & \\
\hline & $\begin{array}{l}\text { Aprendizaje basado en } \\
\text { problemas }\end{array}$ & & \\
\hline
\end{tabular}


La primera acción consiste en identificar una situación de aprendizaje que necesita una mejora. En este caso es una situación muy habitual que afecta a la mayor parte del alumnado, principalmente en asignaturas (o partes de la misma) de carácter teórico. Habitualmente la inactividad del alumnado se relaciona con: disminución de la motivación por el proceso de aprendizaje, aprendizaje débil (por ejemplo, con escasa retención del conocimiento), escaso desarrollo de capacidades cognitivas y un bajo aprendizaje autónomo. Cuanto mayor es la implicación del alumnado en su proceso de aprendizaje, este es más eficaz y eficiente a nivel emocional y cognitivo. Por tanto, "aprender haciendo" mejora el aprendizaje ya que implica mayor número de acciones cognitivas (Dewey, 1916 \& 1929).

En segundo lugar, se identifican modelos de innovación educativa, validados por publicaciones, para conseguir las mejoras buscadas en esa situación de aprendizaje. En la Tabla 1 se presentan, por ejemplo, cinco métodos: Inteligencia colectiva, Micro Flip Teaching, Aprendizaje basado en retos, Aprendizaje servicio, Aprendizaje basado en problemas. Los modelos no suelen ser excluyentes y, por caso, se puede utilizar la Inteligencia colectiva (Fidalgo-Blanco, Sein-Echaluce, \& García-Peñalvo, 2017) junto con Flip Teaching. No obstante, se aconseja al profesorado inexperto en innovación educativa que comience con un método concreto.

El público objetivo en este caso es todo el alumnado de la asignatura, ya que esta situación (la pasividad) suele ser mayoritaria, principalmente en clases magistrales ( $y$ tampoco perjudica a los ya activos).

El impacto suele estar validado en las publicaciones científicas donde se ha incluido el método elegido. En este caso, el impacto podría centrarse en indicadores de actividad tales como: preguntas y respuestas del alumnado al profesorado o entre ellos; debates; reflexiones sobre un determinado concepto; creación de conocimiento por parte del alumnado de forma individual o cooperativa; y compartición del conocimiento entre el alumnado. Asimismo el impacto se puede medir no solo a través de los resultados del aprendizaje sino de acciones: la interacción entre el alumnado, la calidad y utilidad del conocimiento creado por el alumnado o el aumento de la retroalimentación.

Con estos elementos se construye la primera etapa, el módulo de planificación, donde se ha elegido un método concreto para solventar una situación de aprendizaje determinada.

\subsection{Módulo Aplicación}

El módulo Aplicación del método MAIN se basa en identificar los procesos que intervienen en cada modelo de innovación educativa junto con sus relaciones. Conociendo los procesos de los distintos modelos se podrían llegar a combinar, añadir nuevos procesos o sustituir otros. Esto permite llevar a cabo innovación, de forma sencilla, en los modelos de los métodos de innovación educativa. 
Para mayor claridad, en este caso se trabajará con un solo método de innovación educativa, el método Micro Flip Teaching (MFT) (Fidalgo-Blanco, Martínez-Nuñez, Borrás-Gene, \& Sánchez-Medina, 2017; Fidalgo-Blanco, Sein-Echaluce, \& García-Peñalvo, 2018b). En su creación hemos colaborado los autores de este trabajo a partir de la metodología Flip Teaching (FT) o Flipped Classroom y sus resultados han sido validados en relación con el aumento de la participación activa del alumnado (García-Peñavo, Fidalgo-Blanco, Sein-Echaluce, \& Conde, 2016)

Dicho modelo tiene validada la información de la Tabla 1 referida al impacto.

Flip Teaching se caracteriza por la inversión del modelo educativo en cuanto al lugar de realización de dos de las actividades más habituales dentro del proceso de aprendizaje: la lección y los deberes. La inversión del modelo se basa en que la lección se realiza en casa y los deberes se realizan en clase.

A continuación se desarrollan los sub-módulos del módulo Aplicación, es decir, el modelo funcional, la tecnología y la estrategia.

\subsubsection{Modelo funcional}

Habitualmente el modelo se presenta en función de la herramienta a utilizar, de una acción o de ambas. Por ejemplo, la Figura 3 representa el modelo de aplicación del método MFT bajo este punto de vista.

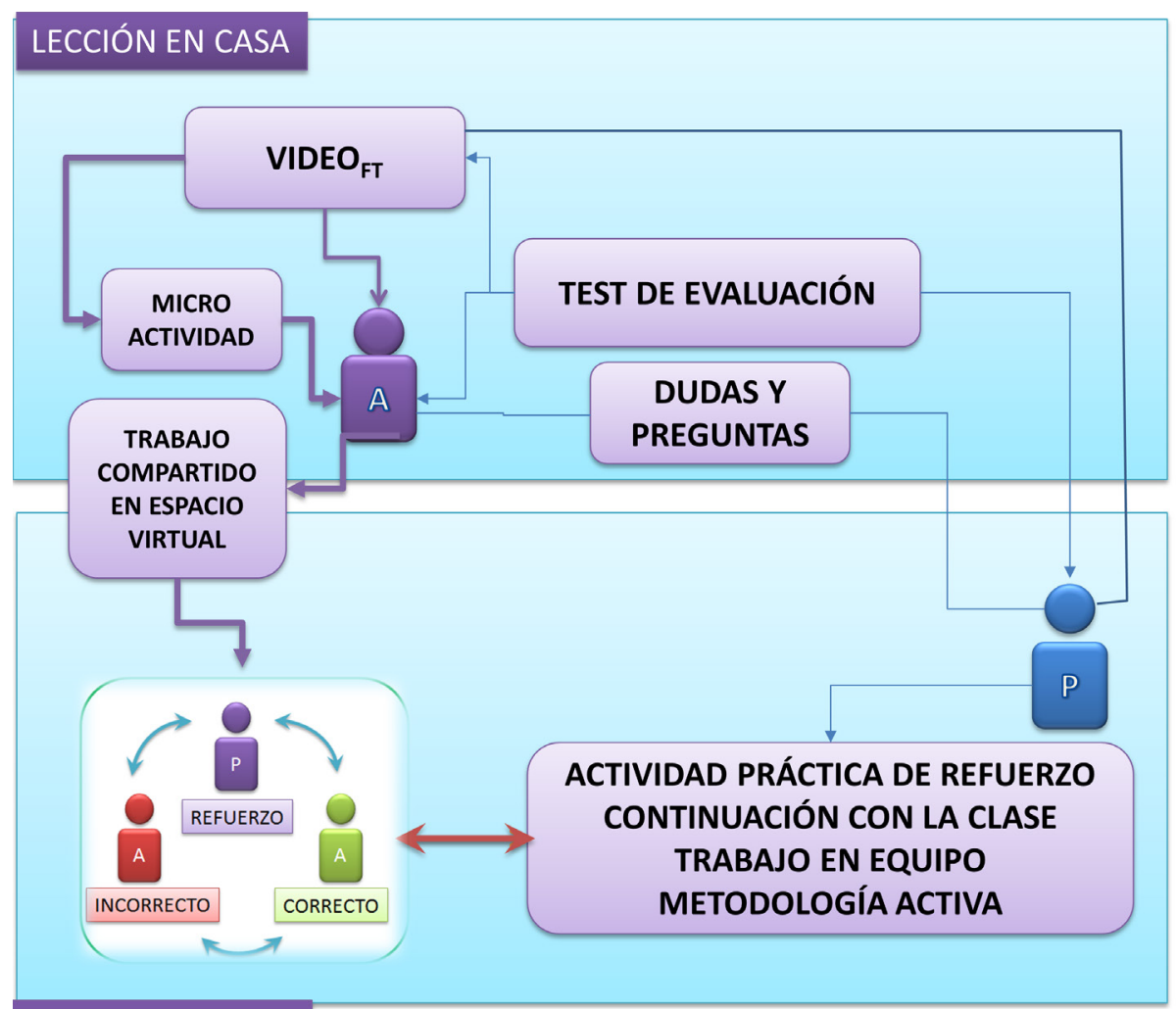

DEBERES EN CLASE 
Esta es una visión clásica del método Flip Teaching en la que el profesorado debe crear videos e incluir foro para dudas y cuestionarios (tests) de evaluación. Además, el alumnado realiza un trabajo (individual o cooperativo) fuera del aula y, ya en el aula, se trabaja con los resultados del mismo, contando con la retroalimentación positiva o negativa del profesorado. Posteriormente, el profesorado continúa la sesión presencial con cualquier metodología activa. La Tabla 2 muestra una visión que se puede obtener a través de dicho modelo. En la primera fila de se presenta la visión principal del método de innovación Flip Teaching y en las siguientes filas las actividades que deben realizar el profesorado y el alumnado.

\begin{tabular}{|l|l|}
\hline Lección en casa & Deberes en clase \\
\hline El alumnado visualiza un video & El alumnado presenta el trabajo \\
\hline El alumnado realiza un cuestionario & Se realiza debate público \\
\hline El alumnado dispone de un foro para dudas & El profesorado participa en el debate \\
\hline El alumnado hace un trabajo & Se continua con la clase presencial \\
\hline
\end{tabular}

Tabla 2. Actividades clásicas a realizar en el método MFT

Esta representación del modelo es sesgada y suele inducir a confusión. Por ejemplo, trabajos de innovación educativa que se presentan bajo el método Flip Teaching consisten simplemente en la realización, por parte del profesorado, de un vídeo y, en el mejor de los casos, su visualización por parte del alumnado.

El método MAIN no trata de identificar las herramientas sino la visión conceptual y funcional de los distintos procesos. La Figura 4 representa el modelo basado en el método MAIN para la Figura 3.

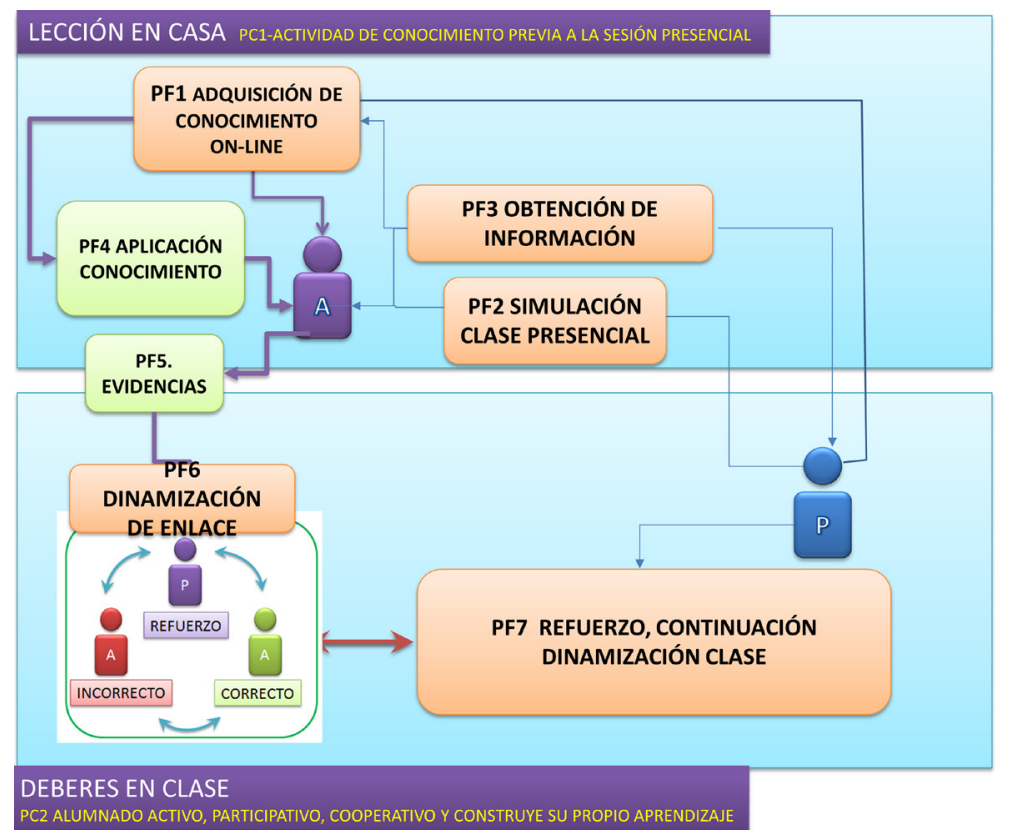

Figura 4. Modelo MAIN para MFT 
El modelo funcional MAIN identifica primeramente procesos conceptuales que definen la innovación, tal y como se muestra en la primera fila de la Tabla 3 (PC1 y PC2), y posteriormente los procesos funcionales, donde se identifica la principal misión del proceso, se incluye en las siguientes filas de la Tabla 3 (PF1, PF2, etc.).

\begin{tabular}{|c|c|}
\hline $\begin{array}{l}\text { Lección en casa. Proceso conceptual: } \\
\text { PC1- Actividad de conocimiento previa a la } \\
\text { sesión presencial }\end{array}$ & $\begin{array}{l}\text { Deberes en clase. Proceso conceptual: } \\
\text { PC2- Participación activa del alumnado }\end{array}$ \\
\hline PF1- Adquisición de conocimiento & $\begin{array}{l}\text { PF6- Dinamización de enlace: utilizar } \\
\text { evidencias de la aplicación del conocimiento } \\
\text { para realizar retroalimentación positiva y } \\
\text { negativa por parte del profesorado }\end{array}$ \\
\hline $\begin{array}{l}\text { PF2- Simulación de la clase presencial. Dotar } \\
\text { al alumnado de los servicios que tiene en una } \\
\text { sesión presencial: resolución de dudas, hablar } \\
\text { con los compañeros y con el profesorado, etc. }\end{array}$ & $\begin{array}{l}\text { PF7- Dinamización de la clase a partir de la } \\
\text { retroalimentación obtenida en el proceso } \\
\text { PF3. Refuerzo a través de micro-lecciones } \\
\text { magistrales }\end{array}$ \\
\hline $\begin{array}{l}\text { PF3- Obtención de información del alumnado. } \\
\text { Retroalimentación tanto para el alumnado } \\
\text { como para el profesorado }\end{array}$ & \\
\hline $\begin{array}{l}\text { PF4- Aplicación de conocimiento a través de } \\
\text { una actividad individual o cooperativa }\end{array}$ & \\
\hline $\begin{array}{l}\text { PF5- Gestión de las evidencias de dicha } \\
\text { aplicación }\end{array}$ & \\
\hline
\end{tabular}

Tabla 3. Procesos conceptuales del método MFT desde el modelo MAIN

Si no se hace este planteamiento del método MAIN, cualquier otro modelo puede conducir a una interpretación muy rígida, como se ha mostrado en la Figura 3. Por ejemplo, es muy habitual que en trabajos de Flip Teaching únicamente se realicen videos, cuando lo importante no es el video sino conseguir que el alumnado participe de forma activa en el aula.

La ventaja que ofrece el modelo basado en el Método MAIN es que facilita la identificación de las actividades a realizar por el profesorado, el alumnado, así como la función de la tecnología. La Tabla 4 expresa esa comparación. 


\begin{tabular}{|c|c|c|}
\hline Actividad & Modelo tradicional & Modelo MAIN \\
\hline $\begin{array}{l}\text { El profesorado } \\
\text { prepara } \\
\text { conocimiento on-line } \\
\text { El alumnado accede } \\
\text { al mismo para su } \\
\text { aprendizaje }\end{array}$ & $\begin{array}{l}\text { El profesorado hace un vídeo. } \\
\text { El alumnado visualiza el vídeo }\end{array}$ & $\begin{array}{l}\text { El profesorado prepara conocimiento } \\
\text { accesible on-line (texto, vídeo, } \\
\text { programa interactivo, sistema } \\
\text { de aprendizaje personalizado, } \\
\text { simulación, problema, etc.) } \\
\text { El alumnado interactúa con ese } \\
\text { conocimiento con diferentes } \\
\text { herramientas tecnológicas, } \\
\text { dependiendo la naturaleza y tipo de } \\
\text { recurso preparado por el profesorado }\end{array}$ \\
\hline
\end{tabular}

Tabla 4. Actividades del modelo MFT basado en MAIN

Por ejemplo, en la segunda columna de la tabla 4 se observa que las principales características del modelo tradicional FT son: "El profesorado hace un video" y "El alumnado visualiza el vídeo". Es decir, el énfasis se pone sobre el recurso, el vídeo, lo que a menudo conduce a la interpretación de que es necesario realizar un video para llevar a cabo la lección en casa durante el método Flip Teaching.

Por otra parte, la visión del método MAIN no pone el énfasis en la herramienta (el video) sino en el proceso a seguir. En este caso (columna 3 de la tabla 4) se da mayor importancia a la adquisición del conocimiento, independientemente del tipo de recurso a utilizar (video, texto, simulación, problema, etc.).

\subsubsection{Tecnología}

En un modelo clásico se suele especificar una tecnología concreta. Por ejemplo, utilizar Moodle para realizar un test de evaluación y así comprobar si el alumnado ha adquirido los conocimientos del video, tal y como se expresa en la Tabla 5.

\begin{tabular}{|c|c|c|}
\hline Componente & Técnica & Herramienta \\
\hline $\begin{array}{c}\text { Test de } \\
\text { evaluación }\end{array}$ & Cuestionario & $\begin{array}{c}\text { Cuestionario } \\
\text { Moodle }\end{array}$ \\
\hline
\end{tabular}

Tabla 5. Tecnología para MFT 
Sin embargo, con el método MAIN la funcionalidad consiste en obtener información que nos permita recabar evidencias del aprendizaje de ese conocimiento. En este caso se identifican técnicas para realizar dicha función y posteriormente las posibles tecnologías, tal y como se expresa en la Tabla 6.

\begin{tabular}{|c|c|c|}
\hline Componente & Técnica & Herramienta \\
\hline $\begin{array}{l}\text { Obtención de } \\
\text { información }\end{array}$ & $\begin{array}{l}\text { Cuestionario / Consulta } \\
\text { / Encuesta/ Rúbrica/ } \\
\text { Análisis de datos de } \\
\text { interacción }\end{array}$ & $\begin{array}{l}\text { Formulario Google / Moodle / Kahoot } \\
\text { / Socrative /Rubistar / Edpuzzle / } \\
\text { Learning Analytics }\end{array}$ \\
\hline
\end{tabular}

Tabla 6. Tecnología para MFT con el método MAIN

La ventaja de no orientar el proceso a una tecnología concreta (frente a la visión conceptual) es que permite identificar múltiples técnicas y las tecnologías disponibles que la hacen posible. Existen habitualmente varias tecnologías para una misma funcionalidad, por tanto se abre un abanico de posibilidades para que el profesorado elija la que más se adapte a sus conocimientos tecnológicos, disponibilidad o preferencias. Así mismo, si una tecnología desaparece y aparece otra más innovadora, entonces puede ser sustituida sin ningún problema. Por ejemplo, una forma muy consolidada de obtener información sobre el aprendizaje es a través de un cuestionario. Sin embargo, en contextos online la interacción del alumnado con la tecnología produce mucha información cuyo análisis permite obtener información del proceso de aprendizaje. Este nuevo enfoque es lo que se denomina Learning Analytics (Siemens, 2012). Así pues, el modelo funcional MAIN es el mismo, independientemente si se utiliza un cuestionario o un sistema de Learning Analytics (Conde, García-Peñalvo, Fidalgo-Blanco, \& Sein-Echaluce, 2017), ya que la función y misión de la tecnología es la misma: obtener información del proceso de aprendizaje del alumnado.

\subsubsection{Estrategia}

El principal problema en las metodologías activas es que el alumnado no tiene hábitos activos y, por tanto, hay que romper una inercia y crear otra nueva más favorable. Así, por lo general, la primera vez que se aplica el método Flip Teaching muy pocas personas han realizado la actividad previa, igual que la segunda vez que se aplica. Esta circunstancia hace que, en ocasiones, el profesorado "obligue" al alumnado a ver el video, por caso, haciendo un examen del mismo en la sesión de aula. Esto no es lo aconsejable ya que se trata de crear hábito activo y cooperativo entre el alumnado y demostrarle que, si participa más activamente, el aprendizaje será más atractivo y con mejores resultados. Hay pues un proceso estratégico para ir creando el hábito entre el alumnado de realizar las actividades previas antes de asistir a clase. 
Este proceso implica que el profesorado cambie su rol clásico durante las primeras sesiones presenciales y que el alumnado también lo cambie. Ninguno de ellos van a realizar los mismos roles que tenían antes de llevar a cabo la innovación educativa.

\subsection{Módulo de Divulgación}

Este módulo está estrechamente relacionado con el anterior. Si se quiere que la experiencia de innovación educativa, además de conseguir mejoras, sea publicable en una revista científica, se deben seguir unos procesos determinados adicionales a los procesos de aprendizaje.

Por ejemplo, la Figura 5 representa tres escenarios científicos de investigación, los más utilizados en las publicaciones científicas, que incluyen experiencias de innovación educativa basadas en el uso de tecnología.

\section{ESCENARIOS DE INVESTIGACIÓN}

\section{LO APLICAS EN TU ASIGNATURA Y NO CONTRASTAS RESULTADOS}

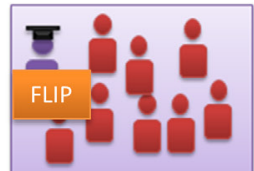

LO APLICAS EN TU ASIGNATURA Y CONTRASTAS RESULTADOS

CON TU ALUMNADO DEL CURSO PASADO
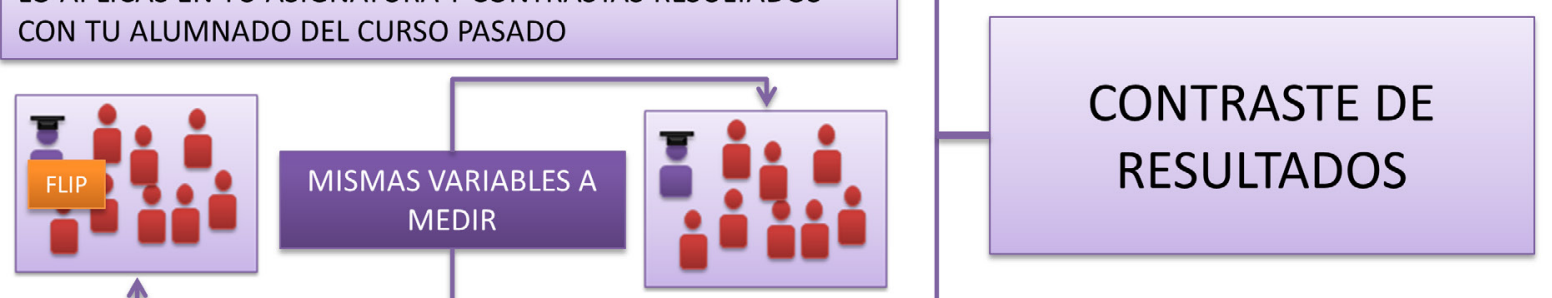

LO APLICAS EN TU ASIGNATURA CON DOS GRUPOS (UNO CON FLIP Y OTRO SIN FLIP)

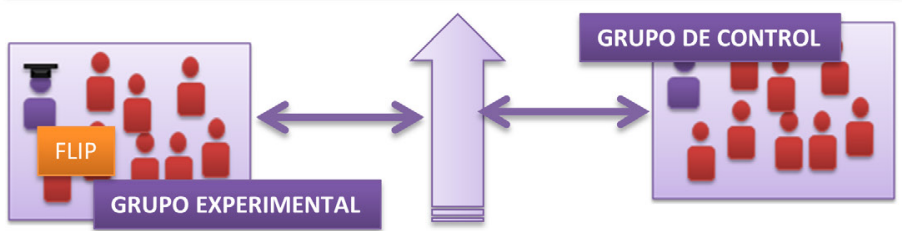

\section{CUASI EXPERIMENTAL}

Figura 5. Escenarios científicos de divulgación de innovación educativa 
Cada uno de los escenarios debe realizar un conjunto de pasos secuenciados en el tiempo, obtener unas evidencias medibles, analizarlas con herramientas validadas y, en base a las mismas, obtener conclusiones. Por ejemplo, y de forma muy resumida, en el método "cuasi experimental" se necesitan evidencias para demostrar que el grupo experimental y el de control son homogéneos y que los procesos de aprendizaje que han realizado cada grupo también son homogéneos para poder comparar los resultados cualitativos y/o cuantitativos obtenidos en cada grupo.

\section{Conclusiones}

El método MAIN está compuesto por un conjunto de módulos y su secuenciación. Algunos de ellos son aportaciones propias del método, como el modelo funcional, mientras que otros se toman de modelos ya consolidados y estandarizados, como el módulo de divulgación científica.

Así mismo, cada módulo se puede utilizar de forma independiente; por ejemplo, se puede utilizar directamente el modelo Aplicación. El modelo funcional descrito para el método MFT es independiente de la situación de aprendizaje donde se vaya a utilizar.

Sin embargo, la utilización de los módulos dentro de la secuencia permite una serie de ventajas frente a la forma actual de realizar la innovación educativa, tales como:

- Centrar la atención en la situación de aprendizaje a mejorar y en los receptores de dicha mejora. Puesto que la educación tiene un conjunto de problemas de aprendizaje que son comunes y generalizados, se pueden identificar y planificar acciones de forma global para su solución. En la sección de resultados se ha mostrado la situación de aprendizaje "alumnado desmotivado", que es una situación habitual en cualquier nivel educativo.

- Identificar y clasificar los métodos de innovación educativa más adecuados o de demostrada eficacia, para solucionar los problemas derivados de esa situación de aprendizaje. Esta característica es sumamente importante, porque si aparece una nueva tendencia de innovación educativa, esta se puede incorporar para mejorar la situación de aprendizaje, añadiendo valor a lo que ya se haya realizado. Habitualmente cuando hay una nueva tendencia en innovación educativa la innovación realizada hasta la fecha se deshecha y se vuelve a comenzar utilizando la nueva tendencia.

- El método MAIN modeliza cada innovación educativa en un conjunto de procesos conceptuales y funcionales, de tal forma que se identifiquen las actividades que debe realizar el alumnado y el profesorado antes de aplicar la innovación educativa. Esto permite tener una idea del esfuerzo, del cambio de rol tanto del alumnado como del profesorado y de la dificultad para implantar esa 
innovación.

- Se puede identificar la misión de la tecnología a utilizar y asociar la misma a cada proceso conceptual. De esta forma se independiza la innovación de la tecnología. Esto permite que el profesorado pueda elegir, en base a distintos criterios, la tecnología a utilizar. Por ejemplo, aquella en la que más experiencia tenga, o de la que disponga el centro donde se va a realizar la innovación educativa o la que le resulte más sencilla de manejar.

- A menudo cuando una tecnología queda obsoleta las innovaciones realizadas con dicha tecnología también quedan obsoletas. El método MAIN es independiente de la evolución tecnológica y si hay una evolución de la misma, el método MAIN permite sustituir la tecnología obsoleta sin que afecte de forma negativa a la innovación.

\section{Agradecimientos}

Agradecemos el apoyo del Servicio de Innovación Educativa de la Universidad Politécnica de Madrid a través del proyecto IE1718.0603 y de la Universidad de Zaragoza, así como a los grupos de investigación LITI (http://www.liti.es) y GIDTIC (http://gidtic.com). Este trabajo ha sido parcialmente financiado por el Ministerio de Economía y Competitividad a través del proyecto DEFINES (Ref. TIN2016-80172-R).

\section{Referencias}

Boyer, E. L. (1990). Scholarship Reconsidered: Priorities of the Professoriate. Princeton, New Jersey: Princeton University Press, The Carnegie Foundation for the Advancement of Teaching. Retrieved from www.josseybass.com.

Conde, M. Á., García-Peñalvo, F. J., Fidalgo-Blanco, Á., \& Sein-Echaluce, M. L. (2017). Can we apply learning analytics tools in challenge based learning contexts? Lecture Notes in Computer Science (including subseries Lecture Notes in Artificial Intelligence and Lecture Notes in Bioinformatics) (Vol. 10296 LNCS). doi:https://doi.org/10.1007/978-3-319-58515-4_19

Dewey, J. (1916). Democracy and education; an introduction to the philosophy of education. New York: The Macmillan Company.

Dewey, J. (1929). Experience and Nature. London: George Allen \& UNWIN, LTD. doi:https://doi. org/10.1037/13377-000

Fidalgo-Blanco, A., Martínez-Nuñez, M., Borrás-Gene, O., \& Sánchez-Medina, J. J. (2017). Micro flip 
teaching - An innovative model to promote the active involvement of students. Computers in Human Behavior, 72. doi:https://doi.org/10.1016/j.chb.2016.07.060

Fidalgo-Blanco, Á., Sein-Echaluce, M. L., \& García-Peñalvo, F. J. (2014). Knowledge spirals in higher education teaching innovation. International Journal of Knowledge Management, 10(4), 16-37. doi:https:// doi.org/10.4018/ijkm.2014100102

Fidalgo-Blanco, Á., Sein-Echaluce, M. L., \& García-Peñalvo, F. J. (2015). Epistemological and ontological spirals: From individual experience in educational innovation to the organisational knowledge in the university sector. Program: Electronic Library and Information Systems, 49(3), 266-288. doi:https:// doi.org/10.1108/PROG-06-2014-0033

Fidalgo-Blanco, Á., Sein-Echaluce, M. L., \& García-Peñalvo, F. J. (2017). Inteligencia colectiva en el aula. Un paradigma cooperativo - [Collective Intelligence in the classroom. A cooperative paradigm]. In La innovación docente como misión del profesorado : Congreso Internacional Sobre Aprendizaje, Innovación y Competitividad (pp. 1-5). Zaragoza: Servicio de Publicaciones Universidad. doi:https://doi. org/10.26754/CINAIC.2017.000001_125

Fidalgo-Blanco, A., Sein-Echaluce, M. L., \& García-Peñalvo, F. (2018a). MAIN. Método de Aplicación de INnovación educativa. doi:https://doi.org/10.5281/ZENODO.1190114

Fidalgo-Blanco, A., Sein-Echaluce, M. L., \& García-Peñalvo, F. J. (2018b). Micro Flip Teaching with Collective Intelligence. In I. A. Zaphiris P. (Ed.), Learning and Collaboration Technologies. LCT 2018. Lecture Notes in Computer Science. Cham: Springer. doi:https://doi.org/10.1007/978-3-319-91743-6_30

García-Peñalvo, F. J. (2015). Mapa de tendencias en Innovación Educativa. Education in the Knowledge Society (EKS), 16(4), 6-23. doi:https://doi.org/10.14201/eks2015164623

García-Peñavo, F. J., Fidalgo-Blanco, A., Sein-Echaluce, M. L., \& Conde, M. A. (2016). Cooperative Micro Flip Teaching. In I. A. Zaphiris P. (Ed.), Learning and Collaboration Technologies. LCT 2016. Lecture Notes in Computer Science (Vol. 9753, pp. 14-24). Springer, Cham. doi:https://doi.org/10.1007/9783-319-39483-1_2

Nonaka, I., \& Takeuchi, H. (1995). The knowledge-creating company: how Japanese companies create the dynamics of innovation. Oxford: Oxford University Press.

Rodríguez-Conde, M. J., Herrera-García, M. E., González-Rogado, A. B., Nieto-Isidro, S., García-Peñalvo, F. J., \& Hernández-Ramos, J. P. (2016). De la Innovación a la Investigación en docencia universitaria (Scholarship of Teaching and Learning, SoTL). Paper presented at the IX Congreso CiDUI 2016 (5-7 de julio de 2016), Barcelona, España. https://goo.gl/i5yf9B 
Sein-Echaluce, M. L., Fidalgo-Blanco, Á., \& Alves, G. (2017). Technology behaviors in education innovation. Computers in Human Behavior, 72, 596-598. doi:https://doi.org/10.1016/j.chb.2016.11.049

Siemens, G. (2012). Learning analytics: envisioning a research discipline and a domain of practice LAK '12 Proceedings of the 2nd International Conference on Learning Analytics and Knowledge (pp. 4-8). New York, NY, USA: ACM. doi:https://doi.org/10.1145/2330601.2330605 